ALEA, Lat. Am. J. Probab. Math. Stat. 18, 707-725 (2021)

DOI: $10.30757 /$ ALEA.v18-26

\title{
Phase transitions for degenerate random environments
}

\author{
Mark Holmes and Thomas S. Salisbury \\ School of Mathematics and Statistics \\ The University of Melbourne. \\ E-mail address: holmes.m@unimelb.edu.au \\ Department of Mathematics and Statistics \\ York University, Toronto. \\ E-mail address: salt@yorku.ca
}

\begin{abstract}
We study a class of models of i.i.d. random environments in general dimensions $d \geq 2$, where each site is equipped randomly with an environment, and a parameter $p$ governs the frequency of certain environments that can act as a barrier. We show that many of these models (including some which are nonmonotone in $p$ ) exhibit a phase transition for the geometry of connected clusters as $p$ varies.
\end{abstract}

\section{Introduction}

Models of (i) random media, and (ii) random walks on them, are important objects of study in modern discrete probability, with a vast literature.

For (i), typically one of the first tasks is to establish the existence of a phase transition as some parameter of the model is varied. In standard percolation models the phase transition often takes the form: there exists a non-trivial critical value $p_{c}$ such that for $p>p_{c}$ there is an infinite connected cluster (and with positive probability the connected cluster of the origin is infinite), while for $p<p_{c}$ there is no infinite cluster. Subsequent analysis focuses e.g. on the size, shape, and finer structure of finite clusters in the different regimes, with the behaviour of the model at the critical point $p_{c}$ being of particular interest. See for example Grimmett (1999).

One of the most fundamental examples of (ii) is the so-called random walk in i.i.d. random environment in $\mathbb{Z}^{d}$, where each site has its own set of transition probabilities for departures from that site, and these are chosen in an i.i.d. fashion

Received by the editors May 8th, 2020; accepted January 26th, 2021.

2010 Mathematics Subject Classification. 60K35, 82B43.

Key words and phrases. Random environment, phase transition, percolation.

The work of MH is supported by Future Fellowship FT160100166 from the Australian Research Council. The work of TS is supported by NSERC and the Fields Institute. 
between different sites. This has been studied for decades, but almost always under the assumption that the environment is (uniformly) elliptic, which means that step probabilities from sites to their nearest lattice neighbours are always strictly positive (see e.g. Bolthausen and Sznitman, 2002; Hughes, 1996; Zeitouni, 2004). By dropping this assumption, fundamental new questions arise, such as whether there are sites that are unreachable by the walk, and whether or not the random walker can (and will) get stuck in a finite region of the lattice (see e.g. Holmes and Salisbury, 2014b, 2017 together with the references in the latter). Both of these are in fact questions about models of random media called degenerate random environments (Holmes and Salisbury, 2014a), which are essentially the random directed graphs one gets from the random environment above by inserting an arrow (directed edge) from a site $x$ to its neighbour $x+e$ if the non-elliptic random environment has assigned positive probability for stepping in direction $e$ from the point $x$. Arguably the situation of most interest is when the random walker does not get stuck on a finite set, and it turns out that there is a simple condition on the degenerate random environment which is equivalent to the random walker almost surely not getting stuck (Holmes and Salisbury, 2014b, Theorem 1.2).

In this paper we consider various degenerate random environments in general dimensions (that satisfy the simple condition mentioned above) that include a certain parameter $p$ that determines the relative frequency of one collection of environments versus another collection. Relative frequencies within each of these two collections are fixed. Canonical examples (such as Example 1.1 below) have just one member in each collection, so the parameter $p$ just determines the relative frequencies of the two possible local environments.

Fix $d \geq 2$, and set $[d]=\{1,2, \ldots, d\}$. Let $\mathcal{E}_{+}=\mathcal{E}_{+}(d)=\left\{e_{i}\right\}_{i \in[d]}$ denote the set of canonical basis vectors for $\mathbb{Z}^{d}$ and let $\mathcal{E}_{-}=\mathcal{E}_{-}(d)=\left\{-e_{i}\right\}_{i \in[d]}$ and $\mathcal{E}=\mathcal{E}(d)=\mathcal{E}_{+}(d) \cup \mathcal{E}_{-}(d)$.

Example 1.1. The orthant model in dimension $d \geq 2$ is the random directed graph in which a vertex $x \in \mathbb{Z}^{d}$ either connects (with probability $p$ ) to each $x+e, e \in \mathcal{E}_{+}$, or (with probability $1-p$ ) to each $x+e, e \in \mathcal{E}_{-}$.

The main contribution of this paper is demonstrating the existence of a nontrivial phase transition for the forward cluster (the set of points $\mathcal{C}_{o}$ reachable from the origin $o \in \mathbb{Z}^{d}$ by following arrows) for each model under consideration. Although the proofs are not inherently difficult, they are not merely applications or modifications of standard percolation arguments. Indeed, the nature of the phase transitions herein is rather different from many other models of random media because in our context $\mathcal{C}_{o}$ will always be infinite (in some cases our "phases" take the form $\mathcal{C}_{o}=\mathbb{Z}^{d}$ and $\mathcal{C}_{o} \neq \mathbb{Z}^{d}$ ). Moreover, many of the most interesting examples (including Example 1.1 above) have forward clusters that are non-monotone in the parameter $p$. Results of this kind have been obtained previously for some 2-dimensional models (including the case $d=2$ of Example 1.1), see Holmes and Salisbury (2014a, 2016), by exploiting a duality with certain (monotone) oriented percolation models. No such simple duality exists in higher dimensions. Nevertheless in this paper our first main result (Theorem 1.9) will relate the forward clusters of non-monotone models with those of some monotone models in a particular way. Our second main result (Theorem 1.11) will exhibit a phase transition for the monotone models, and the two theorems together reveal the existence of phase transitions in our general setting. We then go on to prove related results for the 
backward cluster $\mathcal{B}_{o}$, which is the set of points from which the origin can be reached (by following arrows). Backward and forward clusters can be very different in our setting, as can be seen in Example 1.1 where $\mathcal{B}_{o}$ can be finite or infinite for each $p \in(0,1)$.

Roughly speaking, the phase $\mathcal{C}_{o} \neq \mathbb{Z}^{d}$ corresponds to the setting in which we think it is currently feasible to prove a.s. directional transience (hence also the existence of a deterministic velocity) for random walks in corresponding non-elliptic random environments (see for example Holmes and Salisbury, 2014b). Moreover, we believe that in this phase there exist a deterministic strictly convex cone $\boldsymbol{C}$, a direction $w$, and a random variable $N^{1}$ with exponential tail, such that the forward cluster is contained in $\boldsymbol{C}-N w .^{2}$ This is the "main" condition used in Holmes and Salisbury (2017) to prove an invariance principle and ballisticity for the random walk in random environment.

One may also be interested in such phase transitions as part of the general understanding of random media. In particular, degenerate random environments have features in common with various percolation models such as site percolation, oriented site percolation, first passage percolation etc. They are dual to kinds of surface percolation models, which have been studied in various guises elsewhere in the literature. In the phase $\mathcal{C}_{o} \neq \mathbb{Z}^{d}$ one may ask for a shape theorem (of oriented-percolation type) for e.g. the forward cluster or its boundary. This has been achieved in a special case for large $p$ (Holmes and Salisbury, 2021+), but we expect similar results to hold more generally in this phase (note that our models are only partially oriented, so this is highly non-trivial). One can obtain shape theorems of first passage percolation type (consider the set of sites that can be reached from the origin within $n$ steps, and let $n$ go to $\infty$ ) as well ${ }^{3}$. In the phase $\mathcal{C}_{o}=\mathbb{Z}^{d}$ this should hold in every direction, while in the phase $\mathcal{C}_{o} \neq \mathbb{Z}^{d}$ in some directions only finitely many vertices are reachable from the origin.

1.1. Models and main results. Let $\mu$ be a probability measure on the power set of $\mathcal{E}$. Let $\left(\mathcal{G}_{x}\right)_{x \in \mathbb{Z}^{d}}$ be i.i.d. with law $\mu$. This induces a random directed graph on $\mathbb{Z}^{d}$ - insert arrows from $x$ to each of the vertices $\left\{x+e: e \in \mathcal{G}_{x}\right\}$. As noted earlier, these models are called degenerate random environments - see Holmes and Salisbury (2014a, 2016), and their study lays the foundation for understanding random walks in non-elliptic random environments. In this context the arrows from $x$ represent the possible steps that the walk can take from $x$. The settings that interest us are those $\mu$ for which the random walk in i.i.d. random environment does not get stuck on a finite set. According to Holmes and Salisbury (2014b, Theorem 1.2) the random walker does not get stuck if and only if there exists a mutually orthogonal set of directions $V \subset \mathcal{E}$ such that from every site, at least one of the steps in $V$ is available, i.e. $\mu(\{A \subset \mathcal{E}: A \cap V=\varnothing\})=0$.

Let $\mathcal{C}_{x} \subset \mathbb{Z}^{d}$ denote the set of vertices that can be reached from $x \in \mathbb{Z}^{d}$ by following arrows, as well as the sets $\mathcal{B}_{x}=\left\{y \in \mathbb{Z}^{d}: x \in \mathcal{C}_{y}\right\}$ and $\mathcal{M}_{x}=\mathcal{C}_{x} \cap \mathcal{B}_{x}$. The above condition on $\mu$ is in fact equivalent to the condition that $\mathcal{C}_{x}$ is infinite for every $x$ (see e.g. Holmes and Salisbury, 2014a, Lemma 2.2).

\footnotetext{
${ }^{1}$ With $\boldsymbol{C}, w$, and the law of $N$ all depending on the details of the model.

${ }^{2}$ Added in proof: A recent preprint by $\mathrm{T}$. Beekenkamp proves some results in this direction.

${ }^{3}$ This is currently work in progress with a student.
} 
To state our main results we introduce an explicit probability space (with a particular coupling structure) on which our models are defined. Let $(\Omega, \mathcal{F}, \mathbb{P})$ be a probability space on which $\left(U_{x}\right)_{x \in \mathbb{Z}^{d}},\left(U_{x}^{\prime}\right)_{x \in \mathbb{Z}^{d}}$ are i.i.d. standard uniform random variables. Let $k, \ell \in \mathbb{N}, \boldsymbol{E}=\left(E_{1}, \ldots, E_{k}\right), \boldsymbol{F}=\left(F_{1}, \ldots, F_{\ell}\right)$ with each $E_{i}, F_{j} \subset \mathcal{E}$. Let $\mathcal{D}_{k}=\left\{\left(r_{1}, \ldots, r_{k}\right): r_{i} \geq 0\right.$ for each $i$ and $\left.\sum_{i=1}^{k} r_{i}=1\right\}$. For $p \in[0,1], \boldsymbol{r} \in \mathcal{D}_{k}$, $\boldsymbol{q} \in \mathcal{D}_{\ell}$ and $x \in \mathbb{Z}^{d}$, set

$$
\mathcal{G}_{x}= \begin{cases}E_{i}, & \text { if } U_{x}<p \text { and } U_{x}^{\prime} \in\left[\sum_{j=1}^{i-1} r_{j}, \sum_{j=1}^{i} r_{j}\right) \\ F_{i}, & \text { if } U_{x} \geq p \text { and } U_{x}^{\prime} \in\left[\sum_{j=1}^{i-1} q_{j}, \sum_{j=1}^{i} q_{j}\right) .\end{cases}
$$

We denote the $\mathcal{C}_{x}$ for this model by $\mathcal{C}_{x}(\boldsymbol{E}, \boldsymbol{F}, \boldsymbol{r}, \boldsymbol{q}, p)$. If $k=\ell=1$ then $r_{1}=q_{1}=1$ and we say that the model is 2-valued and we write $\mathcal{C}_{x}\left(E_{1}, F_{1}, p\right)$ for the forward cluster. Sometimes, when there is no ambiguity about which sets $E_{1}$ and $F_{1}$ we are referring to, we will simply write $\mathcal{C}_{x}(p)$.

Let $\underline{E}=\cap_{i=1}^{k} E_{i}$ and $\bar{E}=\cup_{i=1}^{k} E_{i}$, and similarly $\underline{F}=\cap_{i=1}^{\ell} F_{i}$ and $\bar{F}=\cup_{i=1}^{\ell} F_{i}$. Let $\Omega_{+}=\left\{x: U_{x}<p\right\}$ (these are the sites that receive an $E$ environment), and $\Omega_{-}=\left\{x: U_{x} \geq p\right\}$.

Remark 1.2. If $E_{i}^{\prime} \subset E_{i}$ for each $i \in[k]$ and $F_{i}^{\prime} \subset F_{i}$ for each $i \in[\ell]$ then for each $\boldsymbol{r}, \boldsymbol{q}$, and $p$, we have $\mathcal{C}_{o}\left(\boldsymbol{E}^{\prime}, \boldsymbol{F}^{\prime}, \boldsymbol{r}, \boldsymbol{q}, p\right) \subset \mathcal{C}_{o}(\boldsymbol{E}, \boldsymbol{F}, \boldsymbol{r}, \boldsymbol{q}, p)$.

Remark 1.3. The case $k=\ell=1, E_{1}=\mathcal{E}_{+}$and $F_{1}=\mathcal{E}_{-}$is what we have referred to above as the orthant model. The sets $\mathcal{C}_{x}\left(\mathcal{E}_{+}, \mathcal{E}_{-}, p\right)$ are non-monotone in $p$.

When $d=2$, the orthant model was studied in Holmes and Salisbury (2014a) and Holmes and Salisbury (2016). Figure 1.1a shows an example. We henceforth assume the following, which clearly holds for the orthant model.

Condition 1.4. $d \geq 2, e_{1} \in \underline{E}, \bar{E} \subset \mathcal{E}_{+}$, and $\underline{F} \supset \mathcal{E} \backslash \underline{E}$.

Remark 1.2 then implies (assuming Condition 1.4), that

$$
\mathcal{C}_{x}(\underline{E}, \mathcal{E} \backslash \underline{E}, p) \subset \mathcal{C}_{x}(\boldsymbol{E}, \boldsymbol{F}, \boldsymbol{r}, \boldsymbol{q}, p) \subset \mathcal{C}_{x}\left(\mathcal{E}_{+}, \mathcal{E}, p\right) .
$$

Under Condition 1.4, from any site at least one of the arrows in $V=\left\{e_{1},-e_{2}\right\}$ is available ( $e_{1}$ is available if the local environment is $E_{i}$ for some $i$, while $-e_{2}$ is available otherwise), so every $\mathcal{C}_{x}$ contains an infinite self-avoiding path.

Example 1.5. The case $k=\ell=1, E_{1}=\mathcal{E}_{+}$and $F_{1}=\mathcal{E}$ will be referred to as the half-orthant model. It is the "maximal" model satisfying Condition 1.4. Since $E_{1} \subset F_{1}, \mathcal{C}_{o}(p)$ is monotone decreasing in $p$ in this case. Obviously $\mathcal{C}_{o}(0)=\mathbb{Z}^{d}$ and $\mathcal{C}_{o}(1)=\left(\mathbb{Z}_{+}\right)^{d}$. It will turn out that there is a non-trivial phase transition for having $\mathcal{C}_{o}(p)=\mathbb{Z}^{d}$.

See Figure 1.1b for an illustration of the half-orthant model, when $d=2$. Likewise we may compare the following two models, with Figure 1.2 showing part of a realisation of the environment and the cluster $\mathcal{C}_{o}(.5)$ for Examples 1.6 and 1.7 in two dimensions.

Example 1.6. If $k=\ell=1, E_{1}=\left\{e_{1}\right\}$ and $F_{1}=\mathcal{E} \backslash\left\{e_{1}\right\}$ then clearly $\mathcal{C}_{o}(0)=$ $\mathbb{Z}_{-} \times \mathbb{Z}^{d-1}$ and $\mathcal{C}_{o}(1)=\mathbb{Z}_{+} \times\{0\}^{d-1}$ so the sets $\mathcal{C}_{x}(p)$ are non-monotone in $p$.

Example 1.7. Take $k=\ell=1, E_{1}=\left\{e_{1}\right\}$ and $F_{1}=\mathcal{E}$. The sets $\mathcal{C}_{x}(p)$ are monotone in $p$. 


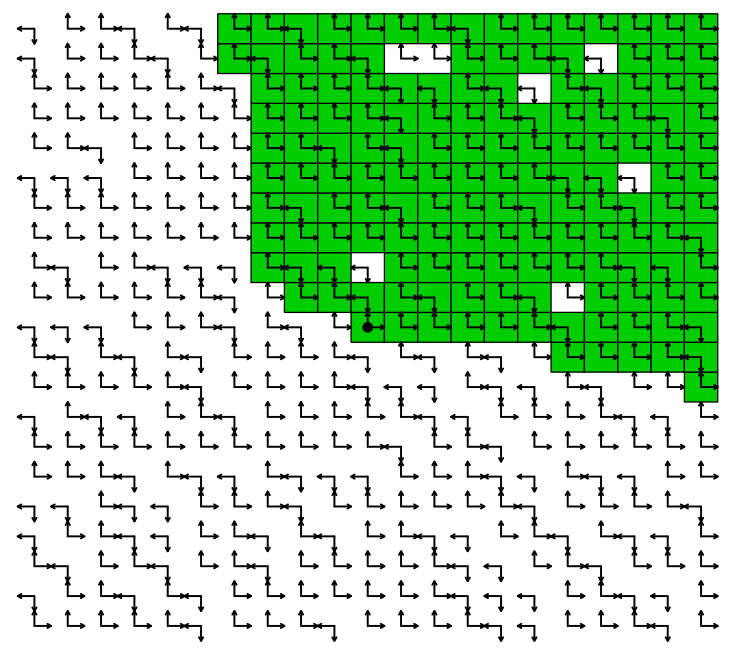

(a) Orthant

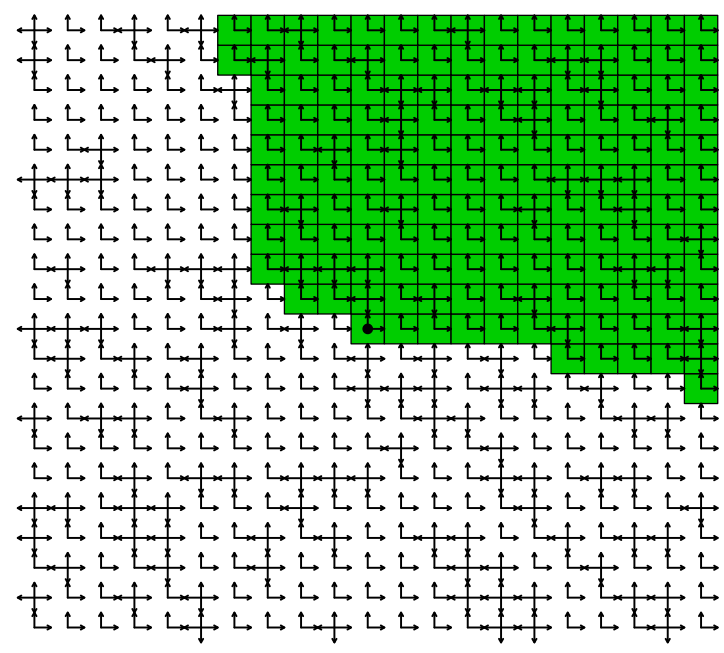

(b) Half-Orthant

Figure 1.1. Realisations of finite parts of the set $\mathcal{C}_{o}$ for the orthant and half-orthant models (Examples 1.1 and 1.5) with $p=0.7$ and $d=2$, and with the origin in the centre. So $k=\ell=1$, and $E_{1}=\left\{e_{1}, e_{2}\right\}$ and $F_{1}=\left\{-e_{1},-e_{2}\right\}$ in (a), while $E_{1}=\left\{e_{1}, e_{2}\right\}$ and $F_{1}=\mathcal{E}(2)$ in (b). They are generated from the same $U$ 's. Note that the boundaries of the two shaded clusters are the same (see Theorem 1.9). 


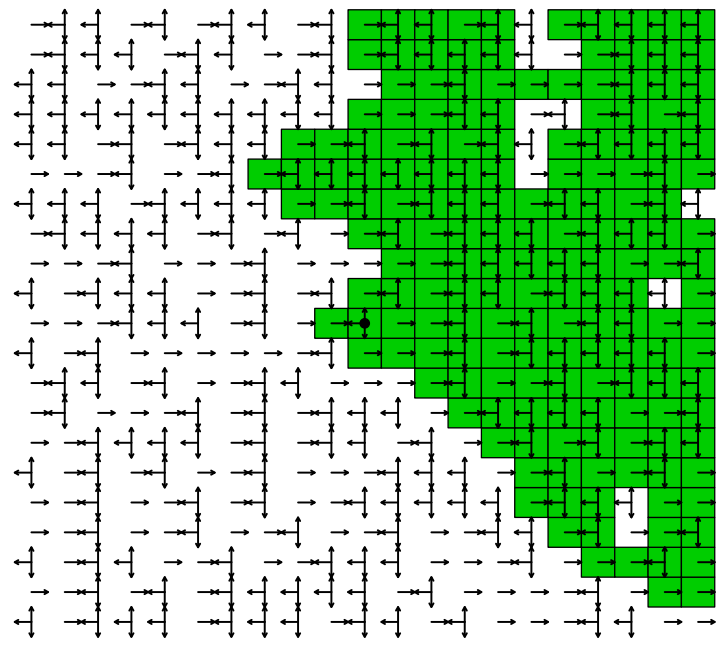

(a) Example 1.6

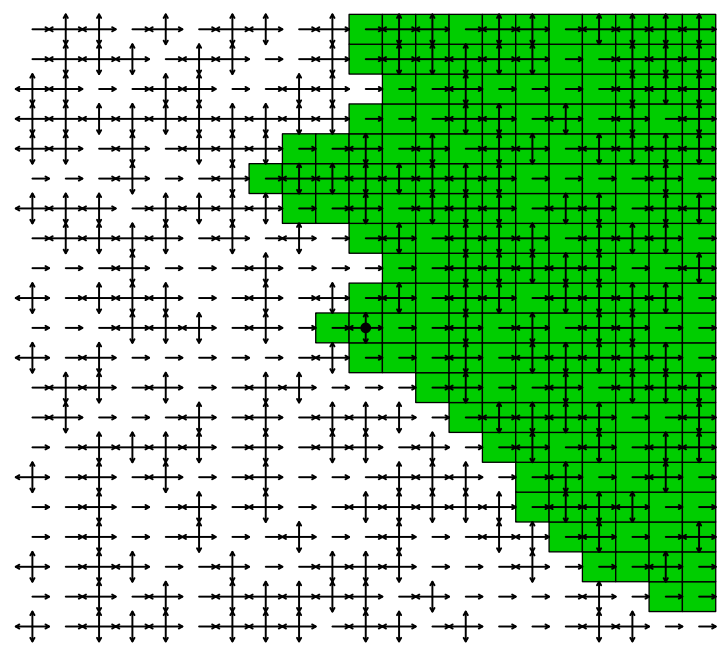

(b) Example 1.7

FIgURE 1.2. Realisations of finite parts of the set $\mathcal{C}_{o}\left(\frac{1}{2}\right)$ for two models with $k=\ell=1$ and $d=2$, and with the origin in the centre. In (a) we have $E_{1}=\left\{e_{1}\right\}$ and $F_{1}=\left\{-e_{1}, e_{2},-e_{2}\right\}$ while in (b) $E_{1}=\left\{e_{1}\right\}$ and $F_{1}=\mathcal{E}(2)$. They are generated from the same environment. Note that the boundaries of the two shaded clusters are the same (see Theorem 1.9).

For fixed $\boldsymbol{F}$, let $\boldsymbol{F}^{*}$ denote the corresponding object with $F_{i}$ replaced with $\mathcal{E}$ for each $i$. Note that we obtain the same model if we take $\ell=1$ and $F_{1}=\mathcal{E}$, so we 
will write $\mathcal{C}_{x}(\boldsymbol{E}, \boldsymbol{r}, p)$ for $\mathcal{C}_{x}\left(\boldsymbol{E}, \boldsymbol{F}^{*}, \boldsymbol{r}, \boldsymbol{q}, p\right)$. Then, by Remark 1.2,

$$
\mathcal{C}_{x}(\boldsymbol{E}, \boldsymbol{F}, \boldsymbol{r}, \boldsymbol{q}, p) \subset \mathcal{C}_{x}(\boldsymbol{E}, \boldsymbol{r}, p) .
$$

For $x \in \mathbb{Z}^{d}$ let $L_{x}:=\inf \left\{k \in \mathbb{Z}: x+k e_{1} \in \mathcal{C}_{o}\right\}$. It is immediate from (1.3) that (writing $L_{x}(\boldsymbol{E}, \boldsymbol{r}, p)$ for $L_{x}\left(\boldsymbol{E}, \boldsymbol{F}^{*}, \boldsymbol{r}, \boldsymbol{q}, p\right)$ )

$$
L_{x}(\boldsymbol{E}, \boldsymbol{F}, \boldsymbol{r}, \boldsymbol{q}, p) \geq L_{x}(\boldsymbol{E}, \boldsymbol{r}, p) .
$$

Remark 1.8. If $x=y+k e_{1}$ for some $k \in \mathbb{Z}$ then $x+j e_{1} \in \mathcal{C}_{o}$ if and only if $y+(k+j) e_{1} \in \mathcal{C}_{o}$, so $L_{y}=L_{x}+k$. It follows that for each $y \in \mathbb{Z}^{d}, L_{y+L_{y} e_{1}}=0$.

For $z \in \mathbb{Z}^{d}$ we define $z_{\{1+\}}=\left\{z+k e_{1}: k \in \mathbb{Z}_{+}\right\}$, and for $A \subset \mathbb{Z}^{d}$

$$
A_{\{1+\}}=\bigcup_{z \in A} z_{\{1+\}}
$$

Our first main result is the following. See Figures 1.1 and 1.2 for 2-valued illustrations when $d=2$. See Figure 1.3 for a simulation of a 3-dimensional model.

Theorem 1.9. Assume Condition 1.4. Then for each $x \in \mathbb{Z}^{d}$, and $p \in(0,1]$,

$$
L_{x}(\boldsymbol{E}, \boldsymbol{F}, \boldsymbol{r}, \boldsymbol{q}, p)=L_{x}(\boldsymbol{E}, \boldsymbol{r}, p) \in[-\infty, \infty) \text { a.s. }
$$

and

$$
\mathcal{C}_{o}(\boldsymbol{E}, \boldsymbol{F}, \boldsymbol{r}, \boldsymbol{q}, p)_{\{1+\}}=\mathcal{C}_{o}(\boldsymbol{E}, \boldsymbol{r}, p) \text { a.s. }
$$

Note that it is not true in general that $\mathcal{C}_{o}(\boldsymbol{E}, \boldsymbol{F}, \boldsymbol{r}, \boldsymbol{q}, p)=\mathcal{C}_{o}(\boldsymbol{E}, \boldsymbol{r}, p)$. However, roughly speaking Theorem 1.9 says that if you only care about the outer boundary of $\mathcal{C}_{o}$ then under Condition 1.4 you may as well set $\ell=1$ and $F_{1}=\mathcal{E}$. Another way of viewing this result is that $\mathcal{C}_{o}(\boldsymbol{E}, \boldsymbol{r}, p)$ is $\mathcal{C}_{o}(\boldsymbol{E}, \boldsymbol{F}, \boldsymbol{r}, \boldsymbol{q}, p)$ with its holes filled in.

The above results reveal that under Condition 1 , a special role is played by the case $\ell=1, F_{1}=\mathcal{E}(d)$. For this reason we will state some results in this special case, i.e. assuming the following condition.

Condition 1.10. $d \geq 2, e_{1} \in \underline{E}, \bar{E} \subset \mathcal{E}_{+}, \ell=1$, and $F_{1}=\mathcal{E}(d)$.

We now state our second main result which reveals a non-trivial phase transition for the occurrence of the event $\left\{\mathcal{C}_{o}=\mathbb{Z}^{d}\right\}$ under Condition 1.10.

Theorem 1.11. Assume Condition 1.10. Then there exists $p_{c}(\boldsymbol{E}, d, \boldsymbol{r}) \in(0,1)$ such that:

$$
\begin{aligned}
& \text { if } p<p_{c} \text { then } \mathcal{C}_{o}(\boldsymbol{E}, \boldsymbol{r}, p)=\mathbb{Z}^{d} \text { almost surely, and } \\
& \text { if } p>p_{c} \text { then } L_{x}(\boldsymbol{E}, \boldsymbol{r}, p) \text { is finite for every } x \in \mathbb{Z}^{d} \text { almost surely (so } \\
& \left.\mathcal{C}_{o}(\boldsymbol{E}, \boldsymbol{r}, p) \neq \mathbb{Z}^{d}\right) .
\end{aligned}
$$

We conjecture that $\mathcal{C}_{o}=\mathbb{Z}^{d}$ in the case $p=p_{c}$ as well.

When $d=2$, Theorem 1.11 follows from Holmes and Salisbury (2016, Propositions 2.3 and 2.4). Those results also imply a version $(d=2$ only) of the Theorem under Condition 1.4, where the conclusion $\mathcal{C}_{o}=\mathbb{Z}^{2}$ (when $p<p_{c}$ ) is replaced with $\overline{\mathcal{C}}_{o}=\mathbb{Z}^{2}$ (when $p<p_{c}$ ), where $\overline{\mathcal{C}}_{o}$ is $\mathcal{C}_{o}$ with its finite "holes" filled in (and note that all of the holes are finite in 2 dimensions). In general dimensions we do not know whether all holes in $\mathcal{C}_{o}$ are finite. Theorems 1.9 and 1.11 seem to be the most natural way of describing the phase transition in general dimensions. The previous versions of Theorems 1.9 and 1.11 in the case $d=2$ made use of a dual percolation 


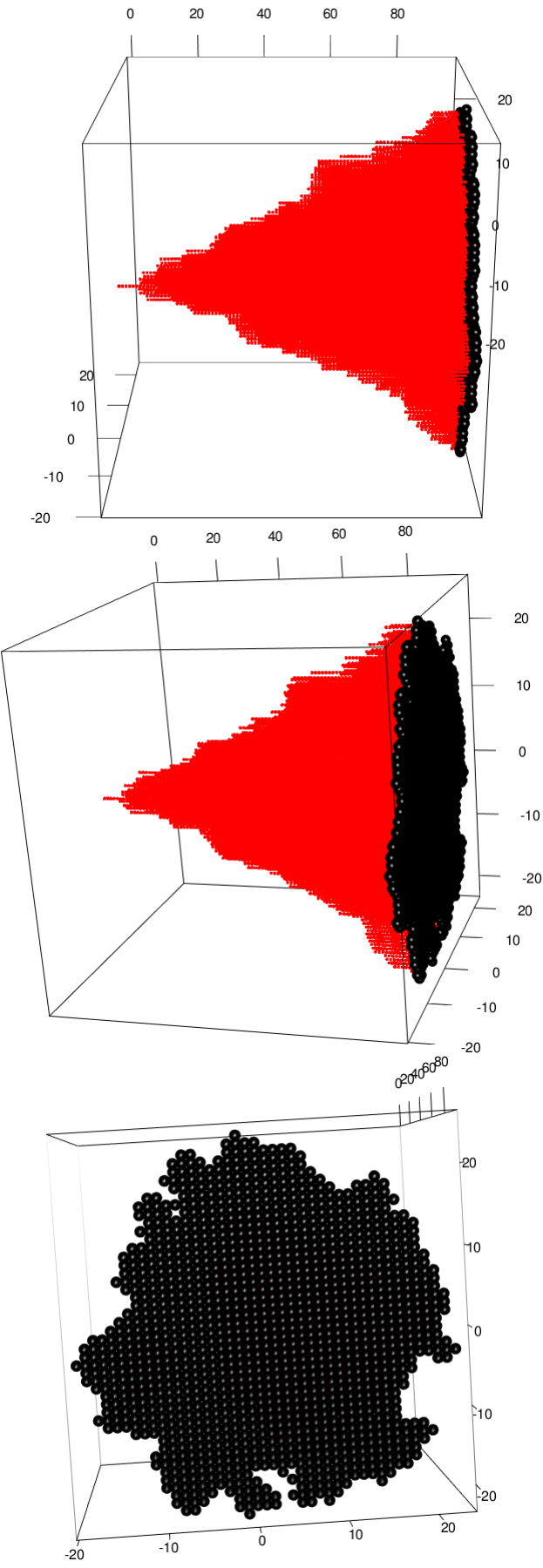

Figure 1.3. A simulation of part of the cluster $\mathcal{C}_{o}(.9)$ for the model with $d=3, k=\ell=1$ and $E_{1}=\left\{e_{1}\right\}$ and $F_{1}=\mathcal{E}(3)$, viewed from 3 different angles. The black/dark vertices are a cross-section where the first coordinate is equal to 100 . 
model. When $d>2$ the corresponding "duality" is with a type of surface percolation. See Grimmett and Holroyd (2010, 2012); Grimmett et al. (2014) for recent work on other higher dimensional percolation structures.

It is natural to ask about asymptotic properties of the boundary of $\mathcal{C}_{o}$ when $p>p_{c}$. To this end, let $\mathbb{P}_{p}$ denote the law of the model with fixed $(\boldsymbol{E}, \boldsymbol{F}, \boldsymbol{r}, \boldsymbol{q}, p)$ and let $Z$ denote the discrete hyperplane $\left\{y \in \mathbb{Z}^{d}: y \cdot e_{1}=0\right\}$.

Open Problem 1.12. Fix $d \geq 2$ and assume Condition 1.4. Prove that if $p>p_{c}$ then for each $v \in Z$ there exists a deterministic $\zeta(v) \in \mathbb{R}$ depending on $\boldsymbol{E}, d, \boldsymbol{r}, \boldsymbol{q}, p$ (but not $\boldsymbol{F}$ ) such that

$$
n^{-1} L_{n v} \rightarrow \zeta(v), \quad \mathbb{P}_{p} \text { - almost surely as } n \rightarrow \infty .
$$

In Holmes and Salisbury $(2021+)$ a version of this result is proved for Examples 1.1 and 1.5 in general dimensions, though with the assumption that $p$ is sufficiently large.

All of the above results concern the forward cluster $\mathcal{C}_{o}$. A crucial difference between forward and backward clusters is that Condition 1.4 does not ensure that $\mathcal{B}_{o}$ is infinite. In the case of Example 1.1, if $e_{i} \in \Omega_{+}$and $-e_{i} \in \Omega_{-}$for each $i \in[d]$ (this has positive probability for any $p \in(0,1)$ ) then there are no arrows pointing to the origin, so $\mathcal{B}_{o}=\{o\}$. Under Condition 1.10 however, $\mathcal{B}_{o}$ will be infinite, since it contains $-\mathbb{Z}_{+} e_{1}$.

Another key difference between forward and backward clusters when $d=2$ is that under Condition 1.4, $\mathcal{B}_{o}$ is "simply connected" as a subset of $\mathbb{Z}^{2}$, while $\mathcal{C}_{o}$ can have holes. The former does not hold for $d>2$ (see Example 3.1 in Section 3). It seems that for $d>2$ there is no simple geometric description of the possible boundaries for finite $\mathcal{B}_{o}$ 's. Infinite $\mathcal{B}_{o}$ clusters appear to be more regular. It would be interesting to characterize infinite clusters that can arise as $\mathcal{B}_{o}$.

For $x \in \mathbb{Z}^{d}$, let $R_{x}=R_{x}(\boldsymbol{E}, \boldsymbol{F}, \boldsymbol{r}, \boldsymbol{q}, p)=\sup \left\{k \in \mathbb{Z}: x+k e_{1} \in \mathcal{B}_{o}\right\}$. The following result shows that under Condition 1.10, the backward and forward clusters have a phase transition at the same point $p_{c}$.

Theorem 1.13. Assume Condition 1.10 and let $p_{c}$ be as in Theorem 1.11. Then if $p<p_{c}$ then $\mathcal{B}_{o}=\mathbb{Z}^{d}$ almost surely, and if $p>p_{c}$ then $R_{x}$ is finite for every $x \in \mathbb{Z}^{d}$.

We conjecture that $\mathcal{B}_{o}=\mathbb{Z}^{d}$ when $p=p_{c}$ as well. The following is an immediate consequence of Theorems 1.11 and 1.13.

Corollary 1.14. Assume Condition 1.10 and let $p_{c}$ be as in Theorem 1.11. Then if $p<p_{c}$ then $\mathcal{M}_{o}=\mathbb{Z}^{d}$ almost surely, and if $p>p_{c}$ then $\mathcal{M}_{o} \neq \mathbb{Z}^{d}$, almost surely.

Open Problem 1.15. Assume Condition 1.10 and let $p_{c}$ be as in Theorem 1.11. Show that when $p>p_{c}, \mathcal{M}_{o}$ is almost surely finite.

Although $\mathcal{B}_{o}$ need not be infinite under Condition 1.4, this condition is sufficient to ensure that $\mathcal{B}_{o}$ is infinite with positive probability (for $p \in(0,1)$ ), and therefore there exist infinite $\mathcal{B}_{x}$ clusters almost surely. To see this, note that the model contains the 2-dimensional model with $\mu\left(\left\{e_{1}\right\}\right)=p$ and $\mu\left(\left\{-e_{1},-e_{2}\right\}\right)=1-p$, for which Holmes and Salisbury (2014a, Proposition 3.4) tells us that $\mathcal{B}_{O}$ is infinite with positive probability. Then the natural analogue of Theorem 1.9 is the following. 
Open Problem 1.16. Assuming Condition 1.4 show that $R_{x}(\boldsymbol{E}, \boldsymbol{F}, \boldsymbol{r}, \boldsymbol{q}, p)=$ $R_{x}(\boldsymbol{E}, \boldsymbol{r}, p)$ almost surely on the event $\left\{\mathcal{B}_{o}(\boldsymbol{E}, \boldsymbol{F}, \boldsymbol{r}, \boldsymbol{q}, p)\right.$ is infinite $\}$.

We will prove a partial result in this direction. For $i \neq 1$ and $z \in \mathbb{Z}^{d}$, define a family of planes $Z^{i}(z)=\left\{x \in \mathbb{Z}^{d}: x^{[k]}=z^{[k]}\right.$ for $\left.k \neq 1, i\right\}$ and the corresponding 2dimensional slices of $\mathcal{B}_{o}$ as $\mathcal{B}_{o}^{i}(z)=Z^{i}(z) \cap \mathcal{B}_{o}$. Call $\mathcal{B}_{o}$ semi-finite if each connected component of each $\mathcal{B}_{o}^{i}(z)$ is finite.

If $\mathcal{B}_{o}=\mathcal{B}_{o}(\boldsymbol{E}, \boldsymbol{F}, \boldsymbol{r}, \boldsymbol{q}, p)$ corresponds to a model satisfying Condition 1.4, write $\mathcal{B}_{o}^{*}=\mathcal{B}_{o}(\boldsymbol{E}, \boldsymbol{r}, p)$ for the corresponding model satisfying Condition 1.10. Note that $\mathcal{B}_{o}^{*}$ is infinite, since $k e_{1} \in \mathcal{B}_{o}^{*}$ for every $k \leq 0$.

Proposition 1.17. Assume Condition 1.4. Then either

(i) $\mathcal{B}_{o}$ is semi-finite, or

(ii) $\mathcal{B}_{o}=\mathcal{B}_{o}^{*}$, or

(iii) $\mathcal{B}_{o}=\left(\mathcal{B}_{o}\right)_{\{1+\}} \neq \mathbb{Z}^{d}$ but $\mathcal{B}_{o}^{*}=\mathbb{Z}^{d}$.

For comparison, in the case of the orthant model with $d=2$, the corresponding alternatives are respectively that $\mathcal{B}_{o}$ is finite; that $\mathcal{B}_{o}$ is either $\mathbb{Z}^{2}$ or the region below a decreasing function; or that $\mathcal{B}_{o}$ is the region above a decreasing function. See Proposition 3.8 of Holmes and Salisbury (2014a) for a more precise statement.

It is trivial that $\mathcal{B}_{o}$ is connected as a subset of the graph $\mathbb{Z}^{d}$. For the complementary cluster, we will show the following.

Proposition 1.18. Assume Condition 1.4. Then either $\mathcal{B}_{o}^{c}$ is empty or $\mathcal{B}_{o}^{c}$ is infinite and connected as a subset of the graph $\mathbb{Z}^{d}$.

Section 3 contains further discussion of related questions. The remainder of this paper is organised as follows. In Section 2 we prove Theorems 1.9 and 1.11. In Section 3 we prove Theorem 1.13 and Propositions 1.17 and 1.18.

\section{Forward Clusters: Proof of Theorems 1.9 and 1.11}

Throughout this section $d \geq 2, \boldsymbol{E}, \boldsymbol{F}, \boldsymbol{r}$, and $\boldsymbol{q}$ as in Condition 1.4 are fixed.

Lemma 2.1. Assume Condition 1.4 and let $p \in(0,1)$. Then

$$
L_{x}<\infty \text { for every } x \in \mathbb{Z}^{d} \text {, a.s. }
$$

Proof: Fix $x \in \mathbb{Z}^{d}$. We will construct a self-avoiding path $P \subset \mathcal{C}_{o}$ from $o$ to an $x_{2}$ of the form $x+k e_{1}$ for some $k \in \mathbb{Z}$. It follows that $L_{x}<\infty$ (possibly $L_{x}=-\infty$ ).

Let $J \subset\{2, \ldots, d\}$ denote the set of indices $j \in\{2, \ldots, d\}$ for which $x \cdot e_{j} \geq 0$ and $e_{j} \in \underline{E}$, and let $J^{\prime}=\{2, \ldots, d\} \backslash J$. If $J=\emptyset$, take $x_{1}=o$. If not, suppose that $J=\left\{j_{1}, \ldots, j_{k}\right\}$ for some $k \geq 1$. Construct a path $P_{1} \subset \mathcal{C}_{o}$ from $o$ as follows: whenever we are at an $F$ site, take the step $-e_{1}$; whenever we are at an $E$ site take the step $e_{j_{1}}$, until the $j_{1}$-st coordinate matches that of $x$, then continue with $j_{2}$ etc. Repeat this until we exhaust the coordinates in $J$. Because all its coordinates are monotonic, $P_{1}$ is self-avoiding, so the environments we see are independent. Therefore we do eventually exhaust the coordinates in $J$, and arrive at a point $x_{1}$ whose $J$ coordinates match those of $x$.

If $J^{\prime}$ is empty then all coordinates of $x_{1}$ (except the first) match those of $x$, and we are done (with $x_{2}=x_{1}$ ). Otherwise from the point $x_{1}$ construct a self-avoiding path $P_{2} \subset \mathcal{C}_{o}$ as follows: if at an $E$ site, take the step $e_{1}$. Otherwise, at an $F$ site, 
take a step that moves some $J^{\prime}$ coordinate closer to the corresponding coordinate of $x$. By definition of $J^{\prime}$, such a step is possible at every $F$ site.

All coordinates of $P_{2}$ are monotonic, so as before, this process eventually terminates at some point point $x_{2} \in \mathcal{C}_{o}$, whose coordinates (other than the first) match those of $x$. Thus $L_{x}<\infty$ as claimed.

Note that $P_{1}$ followed by $P_{2}$ is indeed self-avoiding, despite the fact that the first coordinate initially decreases and then increases, because the last step of $P_{1}$ is never in the direction $-e_{1}$.

Lemma 2.2. Assume Condition 1.4 and let $p \in(0,1)$. Let $x \in \mathbb{Z}^{d}$. Then almost surely on $\left\{L_{x}=-\infty\right\}$, we have $L_{u}=-\infty$ for every $u \in \mathbb{Z}^{d}$.

Proof: Suppose $L_{x}=-\infty$. Then $x-k e_{1} \in \mathcal{C}_{o}$ for infinitely many $k>0$. Suppose $x-k e_{1} \in \Omega_{-} \cap \mathcal{C}_{o}$. Then $x-(k+1) e_{1} \in \mathcal{C}_{o}$ as well. If it $\in \Omega_{-}$then $x-(k+2) e_{1} \in \mathcal{C}_{o}$ as well. We may continue in this way till we find some $x-(k+j) e_{1} \in \Omega_{+} \cap \mathcal{C}_{o}$ (because $p \in(0,1)$ implies that the probability is zero that $x-(k+j) e_{1} \in \Omega_{-}$for every $j>0$ ). In other words, if $x-k e_{1} \in \Omega_{-} \cap \mathcal{C}_{o}$ for infinitely many $k>0$ then also $x-k e_{1} \in \Omega_{+} \cap \mathcal{C}_{o}$ for infinitely many $k>0$.

Similarly one can prove the converse. Thus, on the event that $L_{x}=-\infty$, we have that infinitely many of the points $\left\{x-k e_{1}: k<0\right\}$ are in $\Omega_{+} \cap \mathcal{C}_{o}$ and infinitely many are in $\Omega_{-} \cap \mathcal{C}_{o}$. Using the former, we see that infinitely many of $\left\{x+e-k e_{1}: k<0\right\}$ are in $\mathcal{C}_{o}$, whenever $e \in \underline{E}$. Using the latter, we see that infinitely many of $\left\{x+e-k e_{1}: k<0\right\}$ are in $\mathcal{C}_{o}$, whenever $e \in \mathcal{E} \backslash \underline{E}$. Thus $L_{x+e}=-\infty$ for any $e \in \mathcal{E}$. Using this argument repeatedly proves that $L_{u}=-\infty$ for every $u \in \mathbb{Z}^{d}$.

Under Condition 1.4, this shows that $\left\{L_{o}\right.$ is finite $\}=\left\{L_{x}\right.$ is finite for every $x \in$ $\left.\mathbb{Z}^{d}\right\}$ almost surely. But note that the zero-one law for these events won't be established till later in this section.

Lemma 2.2 can be upgraded slightly (though we will not actually make use of this fact). For $i \in[d]$ and $x \in \mathbb{Z}^{d}$, let $L_{x}^{(i)}=\inf \left\{k \in \mathbb{Z}: x+k e_{i} \in \mathcal{C}_{o}\right\}$, and note that $L_{x}^{(1)}=L_{x}$ by definition. Then we have the following.

Lemma 2.3. Assume Condition 1.4 and let $p \in(0,1)$. Take $x \in \mathbb{Z}^{d}$ and $i \in[d]$. Then almost surely on $\left\{L_{x}^{(i)}=-\infty\right\}$, we have $L_{u}^{(i)}=-\infty$ for every $u \in \mathbb{Z}^{d}$.

Proof: Fix $i \in[d], x \in \mathbb{Z}^{d}$, and $e \in \mathcal{E}$. For $n \in \mathbb{Z}_{+}$, let $M_{n}=\left\{x-k e_{i}: k>n\right\}$. Let $\mathcal{C}_{o}(m)$ denote the set of points $z \in \mathcal{C}_{o}$ for which any shortest path in $\mathcal{C}_{o}$ from $o$ to $z$ is of length $\leq m$. We explore the sets $\mathcal{C}_{o}(m)$ sequentially, starting with $m=0$, for which $\mathcal{C}_{o}(0)=\{o\}$. Clearly $\mathcal{C}_{o}(m+1)$ consists of $\mathcal{C}_{o}(m)$ together with the points we can reach in one step from $\mathcal{C}_{o}(m)$. So $\mathcal{C}_{o}(m+1)$ can be identified just using knowledge of the environments at points in $\mathcal{C}_{o}(m)$.

Given that $N_{r}$ is finite, we define $N_{r+1}=\inf \left\{m: \mathcal{C}_{o}(m) \cap M_{N_{r}} \neq \varnothing\right\}$. If $N_{r+1}$ is finite then we may find some point $y_{r} \in M_{N_{r}} \cap \mathcal{C}_{o}\left(N_{r+1}\right) \backslash \mathcal{C}_{o}\left(N_{r+1}-1\right)$, whose environment has not been explored prior to the iteration $N_{r+1}$. Therefore that environment is independent of what has come before, and we will have $e \in \mathcal{G}_{y_{r}}$ with probability at least $p \wedge(1-p)>0$. If every $N_{r}$ is finite this gives infinitely many independent opportunities to have $e \in \mathcal{G}_{y_{r}}$.

It follows that almost surely, either:

- $N_{r}$ is infinite for some $r$ (in which case $L_{x}^{(i)}>-\infty$ ), or 
- $L_{x}^{(i)}=-\infty$ and $e \in \mathcal{G}_{y_{r}}$ for infinitely many points $y_{r} \in M_{0} \cap \mathcal{C}_{o}$.

The latter case implies that $y_{r}+e \in \mathcal{C}_{o}$ for infinitely many $r$, so $L_{x+e}^{(i)}=-\infty$. Repeating this argument proves the result.

A function $w: \mathbb{Z}^{d} \rightarrow \mathbb{Z}$ is called a side function if for each $y \in \mathbb{Z}^{d}$ and $k \in \mathbb{Z}$,

$$
y+w(y) e_{1}=y+k e_{1}+w\left(y+k e_{1}\right) e_{1} .
$$

In other words, $w$ picks out a single point on each line $y+\mathbb{Z} e_{1}$.

Definition $2.4((\boldsymbol{E},+)$-Barrier $)$. Let $S \subset \mathbb{Z}^{d}$ be a set of points such that

(s1) there exists a side function $w$ such that $S=\left\{y+w(y) e_{1}: y \in \mathbb{Z}^{d}\right\}$,

(s2) $S \subset \Omega_{+}$,

(s3) for $e \in \mathcal{E} \backslash\left\{ \pm e_{1}\right\}$, if $w(y+e)>w(y)$ then for each $k \in[w(y), w(y+e))$, we have $y+k e_{1} \in \Omega_{+}$and $e \notin \mathcal{G}_{y+k e_{1}}$.

For each $y \in \mathbb{Z}^{d}$ and $e \in \mathcal{E} \backslash\left(\underline{E} \cup\left\{-e_{1}\right\}\right)$ define a set $S_{y, e}$ as follows. If $w(y+e)>$ $w(y)$ let $S_{y, e}=\left\{y+k e_{1}: k \in[w(y), w(y+e))\right\}$; otherwise let $S_{y, e}=\varnothing$. Define $\bar{S}=S \cup \bigcup_{y, e} S_{y, e}$. We call any set $\bar{S}$ formed in this way an $(\boldsymbol{E},+$ )-barrier (with side function $w$ ).

Note that taking $k=w(y)$ in (2.2) reveals that $w\left(y+w(y) e_{1}\right)=0$ for each $y \in \mathbb{Z}^{d}$. Therefore (s1) above could be replaced by $S=\left\{x \in \mathbb{Z}^{d}: w(x)=0\right\}$.

Remark 2.5. Note that whether or not a set of points $T \subset \mathbb{Z}^{d}$ is an $(\boldsymbol{E},+)$ barrier can be determined by observing $\left(\mathcal{G}_{z}\right)_{z \in T}$. It also does not depend on $\boldsymbol{F}$. Moreover, if $T$ is an $(\boldsymbol{E},+)$ barrier, then by definition, $T \subset \Omega_{+}$.

On $\left\{L_{x}\right.$ is finite for every $\left.x \in \mathbb{Z}^{d}\right\}$, define $S_{L}=\left\{x+L_{x} e_{1}: x \in \mathbb{Z}^{d}\right\}$. By Remark 1.8 we have $S_{L}=\left\{x \in \mathbb{Z}^{d}: L_{x}=0\right\}$.

Lemma 2.6. Assume Condition 1.4. On $\left\{L_{x}\right.$ is finite for every $\left.x \in \mathbb{Z}^{d}\right\}, \bar{S}_{L}$ is an $(\boldsymbol{E},+)$-barrier with side function $w(y)=L_{y}$ for each $y \in \mathbb{Z}^{d}$, and $w(o) \leq 0$.

Proof: Remark 1.8 shows that $x \mapsto L_{x}$ is a side function. Thus (s1) holds. Now let $z \in S_{L}$. We must have $z \in \Omega_{+}$, since if not then $z-e_{1} \in \mathcal{C}_{o}$, which would contradict the definition of $L_{z}$. This verifies (s2).

Turning to (s3), suppose that $L_{y+e}>L_{y}$ and $e \neq \pm e_{1}$. Then $e \notin \underline{E}$ (since if it were, then $y+L_{y} e_{1} \in \mathcal{C}_{o}$ implies that $y+e+L_{y} e_{1} \in \mathcal{C}_{o}$, and therefore $L_{y} \leq L_{y+e}$ ). Suppose that $y+k e_{1} \in \Omega_{-}$for some $k \in\left[L_{y}, \ldots, L_{y+e}\right)$, and let $\hat{k}$ be the first such $k$. Then $y+\hat{k} e_{1} \in \mathcal{C}_{o}$, since $y+L_{y} e_{1} \in \mathcal{C}_{o}$ and $e_{1} \in \mathcal{G}_{y+j e_{1}}$ for each $j \in\left[L_{y}, \hat{k}\right)$. Therefore $y+\hat{k} e_{1}+e \in \mathcal{C}_{o}$ (since $e \notin \underline{E}$ ), so $L_{y+e} \leq \hat{k}<L_{y+e}$, which is impossible. Therefore $y+k e_{1} \in \Omega_{+}$for each $k \in\left[L_{y}, \ldots, L_{y+e}\right)$.

This also shows that $y+k e_{1} \in \mathcal{C}_{o}$ for each such $k$, so in fact $e \notin \mathcal{G}_{y+k e_{1}}$ for any such $k$ (otherwise $y+k e_{1}+e \in \mathcal{C}_{o}$ and hence $L_{y+e} \leq k$, which is impossible). This verifies (s3), confirming that $\bar{S}_{L}$ is an $(\boldsymbol{E},+)$ barrier.

Finally, we have $w(o) \leq 0$ because $o \in \mathcal{C}_{o} \Rightarrow L_{o} \leq 0$.

Lemma 2.7. Assume Condition 1.10. Whenever $S \subset \mathbb{Z}^{d}$ satisfies (s1)-(s3) of Definition 2.4, with $w(o) \leq 0$, it follows that $L_{x} \geq w(x)$ for every $x \in \mathbb{Z}^{d}$. Moreover $w(x) \leq 0$ for every $x \in \mathcal{C}_{o}$.

Proof: Suppose it isn't true that all $L_{x} \geq w(x)$. Then there exists some $x \in \mathbb{Z}^{d}$ and a $k<w(x)$ such that $z:=x+k e_{1} \in \mathcal{C}_{o}$. Thus $w(z)=w(x)-k>0$. There 
exists a self-avoiding path $y_{0}, y_{1}, \ldots, y_{N}$ in $\mathcal{C}_{o}$ from $y_{0}=o$ to $y_{N}=z$. Let $z_{1}$ be the first location $y$ along this path at which $w(y)>0$. Then $z_{1} \neq o$ since we've assumed that $w(o) \leq 0$. Let $z_{2}$ denote the location immediately preceding $z_{1}$ along this path. Then $w\left(z_{2}\right) \leq 0$ and $z_{1}=z_{2}+e$ for some $e \neq e_{1}$.

We cannot have $e=e_{1}$, since in that case $w\left(z_{1}\right)=w\left(z_{2}\right)-1<0<w\left(z_{1}\right)$, which is impossible.

We cannot have $e=-e_{1}$ either. If it were, then $w\left(z_{2}\right)-1=w\left(z_{1}\right)<0 \leq w\left(z_{2}\right)$, so in fact $w\left(z_{2}\right)=0$. Thus $z_{2} \in S$, so by (s2) we have $z_{2} \in \Omega_{+}$. This implies that $-e_{1} \notin \mathcal{G}_{z_{2}}$ which is impossible, given the definition of $z_{2}$.

Therefore $e \neq \pm e_{1}$. We know that $w\left(z_{1}\right)>0 \geq w\left(z_{2}\right)$, or in other words, $0 \in\left[w\left(z_{2}\right), w\left(z_{2}+e\right)\right)$. By (s3) it follows that $z_{2} \in \Omega_{+}$and $e \notin \mathcal{G}_{z_{2}}$. This is impossible, given the definition of $z_{2}$, which establishes that all $L_{x} \geq w(x)$.

The final conclusion now holds, because if $x \in \mathcal{C}_{o}$ then $w(x) \leq L_{x} \leq 0$.

In the next argument, for simplicity, we will write $L_{x}$ and $L_{x}^{*}$ respectively for the objects $L_{x}(\boldsymbol{E}, \boldsymbol{F}, \boldsymbol{r}, \boldsymbol{q}, p)$ and $L_{x}(\boldsymbol{E}, \boldsymbol{r}, p)$ of Theorem 1.9. Recall that the former corresponds to a model satisfying Condition 1.4, and the latter to a corresponding model satisfying Condition 1.10. We will adopt the same shorthand for other quantities obtained from these model so that, for example, Theorem 1.9 is the statement that $L_{x}=L_{x}^{*}$ and $\left(\mathcal{C}_{o}\right)_{\{1+\}}=\mathcal{C}_{o}^{*}$.

Proof of Theorem 1.9: If $p=1$ the claim is trivial, so assume $p \in(0,1)$. By (1.4), $L_{x}^{*} \leq L_{x}$ for every $x \in \mathbb{Z}^{d}$. Thus if $L_{x}=-\infty$ for every $x$ then there is nothing to prove.

By Lemma 2.2 we may therefore assume that $L_{u}>-\infty$ for every $u \in \mathbb{Z}^{d}$. By Lemma 2.6, $S_{L}$ satisfies (s1)-(s3) and by definition, $L_{o} \leq 0$. By Lemma 2.7 we obtain that $L_{x}^{*} \geq L_{x}$ for every $x \in \mathbb{Z}^{d}$.

Now consider the second assertion. In one direction, the fact that $e_{1} \in \mathcal{G}_{x}^{*}$ for every $x$ implies that $\left(\mathcal{C}_{o}\right)_{\{1+\}} \subset\left(\mathcal{C}_{o}^{*}\right)_{\{1+\}}=\mathcal{C}_{o}^{*}$. In the other direction, let $x \in \mathcal{C}_{o}^{*}$. Then $L_{x}^{*} \leq 0$ so by the first part of the Theorem, also $L_{x} \leq 0$. This implies that $x \in\left(\mathcal{C}_{o}\right)_{\{1+\}}$, and we're done.

Note that probability enters the above arguments only via Lemmas 2.1 and 2.2. Outside of those results, the proofs are purely graph-theoretic. We cannot entirely eliminate probability however. For example, setting $p=0$ in Example 1.1) gives $\left(\mathcal{C}_{o}\right)_{\{1+\}}=\{(i, j): j \leq 0\}$, whereas $\mathcal{C}_{o}^{*}=\mathbb{Z}^{2}$ in this case.

Proof of Theorem 1.11: Assume Condition 1.10 and that $p \in(0,1)$. Consider the following alternatives:

(i) $L_{x}=-\infty$ for every $x \in \mathbb{Z}^{d}$;

(ii) $L_{x}$ is finite for every $x \in \mathbb{Z}^{d}$.

Lemmas 2.1 and 2.2 show that the event that (i) or (ii) holds has probability one.

Lemma 2.6 shows that in case (ii) there exists an $(\boldsymbol{E},+)$ barrier $\bar{S}_{L}$ with $w(o) \leq 0$. On the other hand, if there is an $(\boldsymbol{E},+)$ barrier $\bar{S}$ with $w(o) \leq 0$ then Lemma 2.7 shows that $L_{x} \geq w(x)$ for every $x \in \mathbb{Z}^{d}$. In other words, (ii) and the existence of an $(\boldsymbol{E},+)$ barrier with $w(o) \leq 0$ are equivalent.

The event that there exists an $(\boldsymbol{E},+)$ barrier (somewhere) is translation invariant, and by ergodicity of the environment it follows that the probability that there exists an $(\boldsymbol{E},+)$ barrier is 0 or 1 . If it is 1 , then it follows that the probability that there exists an $(\boldsymbol{E},+)$ barrier with $w(o) \leq n$ increases to 1 as $n \rightarrow \infty$. By 


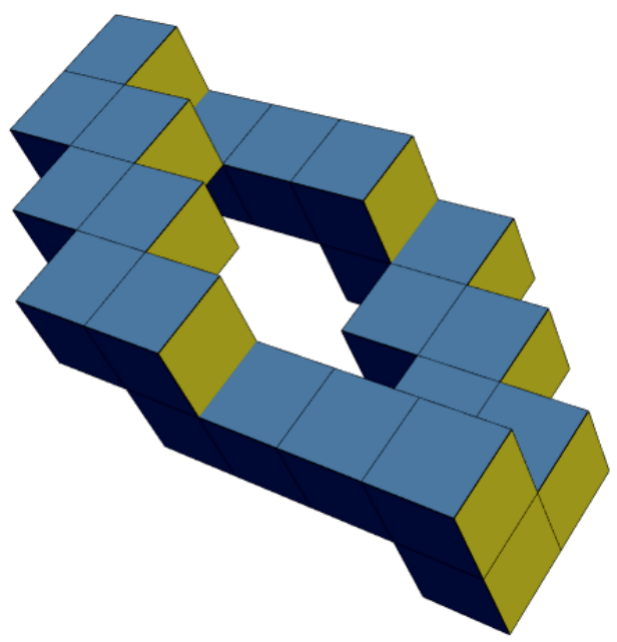

Figure 3.4. A rotation of the irregular (finite) $\mathcal{B}_{o}$ in Example 3.1. In this example neither $\mathcal{B}_{o}$ nor $\mathcal{B}_{o}^{c}$ are simply connected.

translation invariance, this probability does not actually depend on $n$, hence almost surely there is such an $(\boldsymbol{E},+)$ barrier with $w(o) \leq 0$.

We have shown that for each $p \in(0,1)$, either (i) holds almost surely or (ii) holds almost surely. In case (i), since $e_{1}$ is always $\in \mathcal{G}_{x}$ we have $\mathcal{C}_{o}(p)=\mathbb{Z}^{d}$. In case (ii), clearly $\mathcal{C}_{o}(p) \neq \mathbb{Z}^{d}$. Since $\mathcal{C}_{o}(p)$ is monotone decreasing in $p$ this proves the existence of a $p_{c}$ below which $\mathcal{C}_{o}(p)=\mathbb{Z}^{d}$, and above which $\mathcal{C}_{o}(p) \neq \mathbb{Z}^{d}$.

It remains only to show that $p_{c} \in(0,1)$. The fact that $p_{c}>0$ follows because this model dominates $d$-dimensional site percolation (corresponding to setting $E_{i}=\varnothing$ ) with parameter $1-p$. For $1-p$ site percolation the connected cluster of the origin contains infinitely many points of the form $-k e_{1}$ for $k \geq 0$ with positive probability when $1-p$ is larger than the critical probability $p_{c}^{\text {site }}<1$ of the model. It is easy to show that $p_{c}<1$, by counting self-avoiding walks as in e.g. Holmes and Salisbury (2014a, proof of Theorem 4.2).

\section{Backward clusters. Proof of Theorem 1.13}

It is trivial that $\mathcal{B}_{o}$ is connected as a subset of the graph $\mathbb{Z}^{d}$. In Holmes and Salisbury $(2014 \mathrm{a}, 2016)$ for the planar case $d=2$ it is proved that under certain general conditions (implied by Condition 1.4), $\mathcal{B}_{o}$ is simply connected (as a subset of the graph $\mathbb{Z}^{d}$ ). This need not be the case (assuming only Condition 1.4) in higher dimensions as per the following example.

Example 3.1. Consider Example 1.1 in 3 dimensions with $p \in(0,1)$. Then with positive probability neither $\mathcal{B}_{o}$ nor $\mathcal{B}_{o}^{c}$ is simply connected. To be precise, in this model, with positive probability $\mathcal{B}_{O}$ is precisely the loop $(0,0,0),(-1,0,0),(-2,0,0)$, $(-3,0,0),(-3,0,1),(-3,-1,1),(-2,-1,1),(-2,-2,1),(-1,-2,1),(-1,-3,1)$, $(0,-3,1), \quad(0,-3,0), \quad(1,-3,0), \quad(2,-3,0), \quad(3,-3,0), \quad(3,-3,-1), \quad(3,-2,-1)$, $(2,-2,-1),(2,-1,-1),(1,-1,-1),(1,0,-1),(0,0,-1)$ and back to $(0,0,0)$. See Figure 3.4. 
Here we specify whether various (finitely many) vertices $(x, y, z) \in \mathbb{Z}^{3}$ are in $\Omega_{+}$ or $\Omega_{-}$(if the environment isn't specified, it isn't relevant for the example, and * indicates that the vertex is in $\mathcal{B}_{o}$ ).

- The following vertices with $z=0$ are in $\Omega_{-}$:

$$
\begin{aligned}
& (0,0,0)^{*},(-1,0,0)^{*},(-2,0,0)^{*},(3,-3,0)^{*}, \\
& (0,-1,0),(-1,-1,0),(-2,-1,0),(-3,-1,0),(-4,0,0),(-2,-2,0), \\
& (-1,-2,0),(-1,-3,0),(0,-4,0),(1,-4,0),(2,-4,0),(3,-4,0) .
\end{aligned}
$$

- The following vertices with $z=0$ are in $\Omega_{+}$:

$$
\begin{aligned}
& (-3,0,0), 0,-3,0)^{*},(1,-3,0)^{*},(2,-3,0)^{*}, \\
& (0,1,0),(1,0,0),(1,-1,0),(2,-1,0),(0,-2,0),(1,-2,0),(2,-2,0), \\
& (3,-2,0),(4,-3,0),(-1,1,0),(-2,1,0),(-3,1,0) .
\end{aligned}
$$

- The following vertices with $z=-1$ are in $\Omega_{-}$:

$$
\begin{aligned}
& (1,0,-1)^{*},(2,-1,-1)^{*},(3,-2,-1)^{*} \\
& (-3,0,-1),(-2,0,-1),(-1,0,-1),(0,-1,-1),(1,-2,-1), \\
& (0,-3,-1),(1,-3,-1),(2,-3,-1),(3,-4,-1) .
\end{aligned}
$$

- The following vertices with $z=-1$ are in $\Omega_{+}$:

$$
\begin{aligned}
& (0,0,-1)^{*},(1,-1,-1)^{*},(2,-2,-1)^{*},(3,-3,-1)^{*} \\
& (0,1,-1),(1,1,-1),(2,0,-1),(3,-1,-1), \\
& (4,-2,-1),(4,-3,-1) .
\end{aligned}
$$

- The following vertices with $z=1$ are in $\Omega_{-}$:

$$
\begin{aligned}
& (-3,0,1)^{*},(-2,-1,1)^{*},(-1,-2,1)^{*},(0,-3,1)^{*}, \\
& (-4,0,1),(-4,-1,1),(-3,-2,1),(-2,-3,1),(-1,-4,1),(0,-4,1),
\end{aligned}
$$

- The following vertices with $z=1$ are in $\Omega_{+}$:

$$
\begin{aligned}
& (-3,-1,1)^{*},(-2,-2,1)^{*},(-1,-3,1)^{*} \\
& (-3,1,1),(-2,0,1),(-1,0,1),(0,0,1),(-1,-1,1), \\
& (0,-2,1),(1,-3,1),(2,-3,1),(3,-3,1) .
\end{aligned}
$$

- For any vertex $(x, y, 1)^{*}$ appearing above, set $(x, y, 2) \in \Omega_{+}$, and for any vertex $(x, y,-1)^{*}$ appearing above, set $(x, y,-2) \in \Omega_{-}$.

Recall (1.5) and define $z_{\{1-\}}=\left\{z-k e_{1}: k \in \mathbb{Z}_{+}\right\}$, and for $A \subset \mathbb{Z}^{d}$

$$
A_{\{1-\}}=\bigcup_{z \in A} z_{\{1-\}} .
$$

Proof of Theorem 1.13: By Condition 1.10, $e_{1} \in \mathcal{G}_{z}$ for every $z$. Thus $z_{\{1-\}} \subset \mathcal{B}_{z}$ for every $z \in \mathbb{Z}^{d}$. Hence if $z \in \mathcal{B}_{o}$ then $z_{\{1-\}} \subset \mathcal{B}_{o}$.

Suppose that $z \in \mathcal{B}_{o}$ and let $e \neq \pm e_{1}$. Since $p \in(0,1)$ we have that infinitely many points in $(z+e)_{\{1-\}}$ contain $-e$ almost surely, and all of these points are therefore in $\mathcal{B}_{o}$ as well. This proves that $R_{x}>-\infty$ a.s. for every $x \in \mathbb{Z}^{d}$.

If $R_{x}=\infty$ for some $x \in \mathbb{Z}^{d}$ then in fact $x+\mathbb{Z} e_{1} \subset \mathcal{B}_{o}$ and therefore for each $e \neq \pm e_{1}$ almost surely infinitely many points of the form $x+e+k e_{1}$ with $k \geq 0$ 
are in $\mathcal{B}_{o}$ as well. Therefore $R_{x+e}=\infty$ a.s. on $\left\{R_{x}=\infty\right\}$. This proves that \{every $R_{x}$ is finite $\cup$ \{every $\left.R_{x}=\infty\right\}$ has probability one.

If $p>p_{c}$ then with probability 1 there exists an $(\boldsymbol{E},+)$ barrier with $w(o)>0$. Lemma 2.7 shows that if $z \in \mathbb{Z}^{d}$ satisfies $w(z) \leq 0$, then every $x \in \mathcal{C}_{z}$ will satisfy $w(x) \leq 0$. This implies that $o \notin \mathcal{C}_{z}$. Thus no such $z$ can lie in $\mathcal{B}_{o}$, and therefore $R_{x}<w(x)$. So $R_{x}$ is finite for every $x \in \mathbb{Z}^{d}$.

On the other hand, suppose $p<p_{c}$. If $\left(R_{x}\right)_{x \in \mathbb{Z}^{d}}$ are all finite then let $S=$ $\left\{x+\left(R_{x}+1\right) e_{1}: x \in \mathbb{Z}^{d}\right\}$. We claim that $\bar{S}$ is an $(\boldsymbol{E},+)$ barrier, with $w(x)=R_{x}+1$ and $w(o)>0$. But that is a contradiction, since no such barrier exists when $p<p_{c}$. So in fact, all the $R_{x}$ will be infinite. Therefore it only remains to prove that $\bar{S}$ is an $(\boldsymbol{E},+)$ barrier.

To prove this, note first that for each $y \in x+\mathbb{Z} e_{1}$ and $k \in \mathbb{Z}$ we have by definition that $R_{y+k e_{1}}=R_{y}-k$. Therefore $w(x):=R_{x}+1$ is side function, so (s1) is satisfied. Next, $S \subset \Omega_{+}$since for any $z \in S, z-e_{1} \in \mathcal{B}_{o}$ but $z \notin \mathcal{B}_{o}$ so $-e_{1} \notin \mathcal{G}_{z}$. So (s2) holds. Finally, suppose $e \neq \pm e_{1}$ and $w(y+e)>w(y)$. We know that $(y+e)+k e_{1} \in \mathcal{B}_{o}$ for every $k<w(y+e)$, while $y+k e_{1} \notin \mathcal{B}_{o}$ for $k \geq w(y)$. This implies that $e \notin \mathcal{G}_{y+k e_{1}}$ for any $k \in[w(y), w(y+e))$. Therefore also $y+k e_{1} \in \Omega_{+}$for such $k$, which shows (s3).

Before we prove Proposition 1.17 we will state and prove several Lemmas that together will imply the proposition. For $y \in \mathbb{Z}^{d}$ and $k_{1}, k_{2} \in \mathbb{Z}$ with $k_{1}<k_{2}$, let $y\left[k_{1}, k_{2}\right]=\left\{y+k e_{1}: k \in\left[k_{1}, k_{2}\right]\right\}$.

Lemma 3.2. Suppose that for some $y \in \mathbb{Z}^{d}, k_{1}<k_{2} \in \mathbb{Z}$ and $e \in \mathcal{E} \backslash\left\{ \pm e_{1}\right\}$ we have $y\left[k_{1}, k_{2}\right] \subset \mathcal{B}_{o}$ and $\left\{y+k_{1} e_{1}+e, y+k_{2} e_{1}+e\right\} \subset \mathcal{B}_{o}$. Then $(y+e)\left[k_{1}, k_{2}\right] \subset \mathcal{B}_{o}$.

Proof: Either $-e \in \underline{E}$ or $-e \in \underline{F}$. In the first case we have that $(y+e)\left[k_{1}, k_{2}\right] \cap \Omega_{+} \subset$ $\mathcal{B}_{o}$, and since $-e_{1} \in \underline{F}$ it then follows that $(y+e)\left[k_{1}, k_{2}\right] \subset \mathcal{B}_{o}$. In the second case we have that $(y+e)\left[k_{1}, k_{2}\right] \cap \Omega_{-} \subset \mathcal{B}_{o}$, and since $e_{1} \in \Omega_{+}$it follows that $(y+e)\left[k_{1}, k_{2}\right] \subset \mathcal{B}_{o}$.

For $y \in \mathbb{Z}^{d}$, let $y[\mathbb{Z}]=y+\mathbb{Z}_{1}, y\left[\mathbb{Z}_{+}\right]=y+\mathbb{Z}_{+} e_{1}$, and $y\left[\mathbb{Z}_{-}\right]=y+\mathbb{Z}_{-} e_{1}$.

Lemma 3.3. Almost surely, if there exists $y \in \mathbb{Z}^{d}$ such that $y[\mathbb{Z}] \subset \mathcal{B}_{o}$ then $\mathcal{B}_{o}=$ $\mathbb{Z}^{d}$.

Proof: Let $e \in \mathcal{E} \backslash\left\{-e_{1}, e_{1}\right\}$. It suffices to show that $(y+e)[\mathbb{Z}] \subset \mathcal{B}_{o}$.

Either $-e \in \underline{E}$ or $-e \in \underline{F}$. Since $p \in(0,1)$ we have that infinitely many points $z$ in $(y+e)\left[\mathbb{Z}_{+}\right]$have $-e \in \overline{\mathcal{G}}_{z}$ and likewise, infinitely many points $z$ in $(y+e)\left[\mathbb{Z}_{-}\right]$ have $-e \in \mathcal{G}_{z}$. Each such point is therefore in $\mathcal{B}_{o}$. It follows from Lemma 3.2 that $(y+e)[\mathbb{Z}] \subset \mathcal{B}_{o}$ as claimed.

Lemma 3.4. Suppose that there exists $y \in \mathbb{Z}^{d}$ such that $y\left[\mathbb{Z}_{-}\right] \subset \mathcal{B}_{o}$, but $y[\mathbb{Z}] \not \subset \mathcal{B}_{o}$. Then almost surely for every $x \in \mathbb{Z}^{d}$ there exists $K_{x} \in \mathbb{Z}$ such that $\mathcal{B}_{o} \cap x[\mathbb{Z}]=$ $\left(x+K_{x}\right)\left[\mathbb{Z}_{-}\right]$.

Proof: Let $e \in \mathcal{E} \backslash\left\{ \pm e_{1}\right\}$. Since $y\left[\mathbb{Z}_{-}\right] \subset \mathcal{B}_{o}$ and $-e \in \underline{E} \cup \underline{F}$ we have that infinitely many points in $(y+e)\left[\mathbb{Z}_{-}\right]$are also in $\mathcal{B}_{o}$. It follows from Lemma 3.2 that $\left(y+e+k e_{1}\right)\left[\mathbb{Z}_{-}\right] \subset \mathcal{B}_{o}$ for some $k \in \mathbb{Z}$. Repeating this argument as needed proves that for every $x \in \mathbb{Z}^{d}$ there exists $k_{x} \in \mathbb{Z}$ such that $\left(x+k_{x} e_{1}\right)\left[\mathbb{Z}_{-}\right] \subset \mathcal{B}_{o}$. Since $y[\mathbb{Z}] \not \subset \mathcal{B}_{o}$, Lemma 3.3 tells us that there is a largest such $k_{x}$, which we denote by $K_{x}$. 
If there was any $k>K_{x}$ such that $x+k e_{1} \in \mathcal{B}_{o}$ then Lemma 3.2 would imply that $\left(x+k e_{1}\right)\left[\mathbb{Z}_{-}\right] \subset \mathcal{B}_{o}$. This would contradict the definition of $K_{x}$, so in fact $\mathcal{B}_{o} \cap x[\mathbb{Z}]=\left(x+K_{x}\right)\left[\mathbb{Z}_{-}\right]$.

The proof of the following is similar, and is left to the reader.

Lemma 3.5. Suppose that there exists $y \in \mathbb{Z}^{d}$ such that $y\left[\mathbb{Z}_{+}\right] \subset \mathcal{B}_{o}$ but $y[\mathbb{Z}] \not \subset \mathcal{B}_{o}$. Then almost surely for every $x \in \mathbb{Z}^{d}$ there exists $K_{x}^{\prime} \in \mathbb{Z}$ such that $\mathcal{B}_{o} \cap x[\mathbb{Z}]=$ $\left(x+K_{x}^{\prime}\right)\left[\mathbb{Z}_{+}\right]$.

Obviously, under the assumptions of Lemma 3.4 we have $K_{x+e_{1}}=K_{x}-1$, hence $w(x):=K_{x}+1$ satisfies (2.2). Similarly, in Lemma 3.5 we have $K_{x+e_{1}}^{\prime}=K_{x}^{\prime}-1$.

Recall the notation $Z^{i}(z)$ and $\mathcal{B}_{o}^{i}(z)$ given prior to the statement of Proposition 1.17 .

Lemma 3.6. Let $i \neq 1$ and $z \in \mathbb{Z}^{d}$. Then the set $Z^{i}(z) \backslash \mathcal{B}_{o}$ is connected.

Suppose that $B$ is an infinite connected component of $\mathcal{B}_{o}^{i}(z)$. Then for any $z^{\prime} \in$ $Z^{i}(z)$ the set

$$
I=I\left(z^{\prime}\right):=z^{\prime}[\mathbb{Z}] \backslash B,
$$

is a single interval (which is possibly empty or infinite, but not bi-infinite).

Proof: For $y_{1}, y_{2} \in Z^{i}(z) \backslash \mathcal{B}_{o}$, we can follows paths consistent with the environment and consisting of only steps $e_{1}$ (from $\Omega_{+}$sites) and $-e_{i}$ (from $\Omega_{-}$sites) that eventually intersect (as in Proposition 3.8 of Holmes and Salisbury, 2014a). These paths lie entirely in $Z^{i}(z) \backslash \mathcal{B}_{o}$ since only moves $e_{1},-e_{i}$ were used and $y_{1}, y_{2} \in Z^{i}(z) \backslash \mathcal{B}_{o}$. This proves the first claim.

For the second claim, suppose that $I$ is not an interval. Then there exist $y_{1}, y_{2} \in I$ with $y_{1}^{[1]}<y_{2}^{[1]}-1$ and such that $v \in B$ for every $v \in z^{\prime}[\mathbb{Z}]$ with $y_{1}^{[1]}<v^{[1]}<y_{2}^{[1]}$. Then $y_{1}, y_{2} \notin \mathcal{B}_{o}$, since they neighbour $B$ but are $\notin B$. From $y_{1}$ and $y_{2}$ we may follow paths consistent with the environment using only $e_{1}$ and $-e_{i}$ moves from $\Omega_{+}$sites and $\Omega_{-}$sites respectively. These paths eventually meet (again, as in Proposition 3.8 of Holmes and Salisbury, 2014a) and are contained in $Z^{i}(z) \backslash \mathcal{B}_{o}$. Similarly, if $e_{i} \in \underline{E}$ then from $y_{1}$ and $y_{2}$ we may follow paths consistent with the environment using only $-e_{1}$ and $e_{i}$ moves, from $\Omega_{-}$sites and $\Omega_{+}$sites respectively. If $e_{i} \notin \underline{E}$ then we may instead follow paths using only $e_{i}$ and $e_{1}$ moves, from $\Omega_{-}$sites and $\Omega_{+}$sites respectively. In either case the two paths intersect and are contained in $\mathcal{B}_{o}^{c}$.

It follows that each $v$ as above is enclosed by a circuit in $Z^{i}(z) \backslash \mathcal{B}_{o}$ and hence $v$ is not in an infinite component of $\mathcal{B}_{o} \cap Z^{i}(z)$, contradicting that $v \in B$. This shows that $I$ is indeed an interval.

It remains only to prove that $I\left(z^{\prime}\right) \neq z^{\prime}[\mathbb{Z}]$. If $\mathcal{B}_{o}=\mathbb{Z}^{d}$ then this holds, since $I\left(z^{\prime}\right)=\emptyset$. So assume this is not the case. Since $B$ is non-empty there is some $u \in \mathbb{Z}^{d}$ such that $u[\mathbb{Z}] \cap B$ is non-empty, and since $I(u)$ is an interval, $u[\mathbb{Z}] \cap B$ must contain a half line. Without loss of generality it is $y\left[\mathbb{Z}_{-}\right]$for some $y \in u[\mathbb{Z}]$. Because $\mathcal{B}_{o} \neq \mathbb{Z}^{d}$, Lemma 3.3 implies that $y[\mathbb{Z}] \not \subset B_{o}$. So by Lemma 3.4, for every $x \in Z^{i}(z)$ there is a $K_{x} \in \mathbb{Z}$ such that $\mathcal{B}_{o} \cap x[\mathbb{Z}]=\left(x+K_{x} e_{1}\right)\left[\mathbb{Z}_{-}\right]$. But $\bigcup_{x \in Z^{i}(z)}\left(x+K_{x} e_{1}\right)\left[\mathbb{Z}_{-}\right]$ is connected in $Z^{i}(z)$ and intersects $B$ (just take $x=u$ ), so in fact it is equal to $B$. Thus $B$ intersects $z^{\prime}[\mathbb{Z}]$, so $I\left(z^{\prime}\right) \neq z^{\prime}[\mathbb{Z}]$.

Proof of Proposition 1.1\%: If $v[\mathbb{Z}] \subset \mathcal{B}_{o}$ for some $v \in \mathbb{Z}^{d}$ then $\mathcal{B}_{o}=\mathbb{Z}^{d}$ by Lemma 3.3, and we are in case (ii). So assume this is not the case. 
If all components of $\mathcal{B}_{o}^{i}(z)$ are finite for all $i$ and $z$, then $\mathcal{B}_{o}$ is semi-finite, and we are in case (i).

Otherwise, for some $i \neq 1$ and $z \in \mathbb{Z}^{d}$ the set $\mathcal{B}_{o}^{i}(z)$ has an infinite component $B$. Without loss of generality we assume $i=2$. By Lemma 3.6, for each $z^{\prime} \in Z^{2}(z)$ we have that $I\left(z^{\prime}\right)=z^{\prime}[\mathbb{Z}] \backslash B$ is an interval that is not bi-infinite, so there exists $z^{\prime \prime} \in \mathbb{Z}\left(z^{\prime}\right)$ such that either $z^{\prime \prime}\left[\mathbb{Z}_{-}\right] \subset B$ or $z^{\prime \prime}\left[\mathbb{Z}_{+}\right] \subset B$.

In the second case, since $z^{\prime \prime}\left[\mathbb{Z}_{+}\right] \subset B \subset \mathcal{B}_{o}$ but $z^{\prime \prime}[\mathbb{Z}] \not \subset \mathcal{B}_{o}$, by Lemma 3.5 we have that each $\mathcal{B}_{o} \cap x[\mathbb{Z}]$ has the form $\left(x+K_{x}^{\prime}\right)\left[\mathbb{Z}_{+}\right]$for some $K_{x}^{\prime} \in \mathbb{Z}$. It follows that $\mathcal{B}_{o}=\left(\mathcal{B}_{o}\right)_{\{1+\}}$ and $\mathcal{B}_{o}^{*}=\mathbb{Z}^{d}$, so case (iii) of the Proposition holds.

In the first case, Lemma 3.4 implies that for every $x, \mathcal{B}_{o} \cap x[\mathbb{Z}]$ has the form $\left(x+K_{x}\right)\left[\mathbb{Z}_{-}\right]$for each some $K_{x} \in \mathbb{Z}$. Let $S=\left\{x+\left(K_{x}+1\right) e_{1}: x \in \mathbb{Z}^{d}\right\}$. Since $-e_{1} \notin \mathcal{G}_{x+\left(K_{x}+1\right) e_{1}}$ by definition of $K_{x}$ we have that (s1) and (s2) of Definition 2.4 hold for $S$ (with $w(x):=K_{x}+1$ ). Suppose that $w(y+e)>w(y)$, and let $J=$ $[w(y), w(y+e))$. Then $e \notin \cup_{j \in J} \mathcal{G}_{y+j e_{1}}$, so either $\left\{y+j e_{1}: j \in J\right\} \subset \Omega_{+}($if $e \notin \underline{E})$ or $\left\{y+j e_{1}: j \in J\right\} \subset \Omega_{-}$(if $e \in \underline{E}$ ).

The second alternative cannot occur, since if it did then $-e_{1} \in \mathcal{G}_{y+j e_{1}}$ for each $j \in J$, so $\left\{y+j e_{1}: j \in J\right\} \subset \mathcal{B}_{o}$ which contradicts the definition of $w(y)$. Therefore $\left\{y+j e_{1}: j \in J\right\} \subset \Omega_{+}$, so (s3) of Definition 2.4 holds, i.e. $\bar{S}$ is an $(\boldsymbol{E},+)$ barrier with side function $w$. It remains an $(\boldsymbol{E},+)$ barrier with side function $w$ when we change all $\Omega_{-}$sites to $\mathcal{E}$, so this proves that $\mathcal{B}_{o}=\mathcal{B}_{o}^{*}$ in this case.

Proof of Proposition 1.18: If $\mathcal{B}_{o}^{c}$ is non-empty then there exists $y \in \mathcal{B}_{o}^{c}$, and since $\mathcal{C}_{y} \subset \mathcal{B}_{o}^{c}$ we conclude that $\mathcal{B}_{o}^{c}$ is infinite.

To show connectedness, let $y_{1}, y_{2} \in \mathcal{B}_{o}^{c}$. We will construct self-avoiding paths from each, consistent with the environment, that eventually meet. By definition, both paths must lie in $\mathcal{B}_{o}^{c}$, which will establish the result.

Without loss of generality, $y_{1}^{[d]} \leq y_{2}^{[d]}$. Build a path from $y_{2}$ by following $e_{1}$ at sites in $\Omega_{+}$and $-e_{d}$ at sites in $\Omega_{-}$till we reach a point $y_{2}^{\prime} \in \mathcal{B}_{o}^{c}$ whose $d^{\prime}$ th coordinate agrees with that of $y_{1}$. Let $y_{1}^{\prime}=y_{1}$. Repeating the same argument, now starting from $y_{1}^{\prime}$ and $y_{2}^{\prime}$, we will in turn reach points whose $d^{\prime}$ th and $(d-1)$ 'st coordinates agree. Continuing in this way, we'll reach points $x_{1}, x_{2} \in \mathcal{B}_{o}^{c}$, all of whose coordinates agree, other than the first two. In the notation from before Proposition 1.17 we'll have that $x_{1}, x_{2}$ belong to the plane $Z^{2}\left(x_{1}\right)$.

But from $x_{1}$ and $x_{2}$ we may now apply the planar construction of Proposition 3.8 of Holmes and Salisbury (2014a) (also used above in the proof of Lemma 3.6) to build paths in $Z^{2}\left(x_{1}\right) \cap \mathcal{B}_{o}^{c}$ that eventually cross. Thus $y_{1}$ and $y_{2}$ both connect to that crossing point.

Acknowledgements. The authors thank an anonymous referee for several suggestions which helped improve the paper.

\section{References}

Bolthausen, E. and Sznitman, A.-S. Ten lectures on random media, volume 32 of DMV Seminar. Birkhäuser Verlag, Basel (2002). ISBN 3-7643-6703-2. MR1890289. 
Grimmett, G. Percolation, volume 321 of Grundlehren der Mathematischen Wissenschaften [Fundamental Principles of Mathematical Sciences]. Springer-Verlag, Berlin, second edition (1999). ISBN 3-540-64902-6. MR1707339.

Grimmett, G. R. and Holroyd, A. E. Plaquettes, spheres, and entanglement. Electron. J. Probab., 15, 1415-1428 (2010). MR2721052.

Grimmett, G. R. and Holroyd, A. E. Geometry of Lipschitz percolation. Ann. Inst. Henri Poincaré Probab. Stat., 48 (2), 309-326 (2012). MR2954256.

Grimmett, G. R., Holroyd, A. E., and Kozma, G. Percolation of finite clusters and infinite surfaces. Math. Proc. Cambridge Philos. Soc., 156 (2), 263-279 (2014). MR3177869.

Holmes, M. and Salisbury, T. S. Degenerate random environments. Random Structures Algorithms, 45 (1), 111-137 (2014a). MR3231085.

Holmes, M. and Salisbury, T. S. Random walks in degenerate random environments. Canad. J. Math., 66 (5), 1050-1077 (2014b). MR3251764.

Holmes, M. and Salisbury, T. S. Forward clusters for degenerate random environments. Combin. Probab. Comput., 25 (5), 744-765 (2016). MR3531440.

Holmes, M. and Salisbury, T. S. Conditions for ballisticity and invariance principle for random walk in non-elliptic random environment. Electron. J. Probab., 22, Paper No. 81, 18 (2017). MR3710801.

Holmes, M. and Salisbury, T. S. A shape theorem for the orthant model $(2021+)$. To appear in Ann. Probab.

Hughes, B. D. Random walks and random environments. Vol. 2, Random environments. Oxford Science Publications. The Clarendon Press, Oxford University Press, New York (1996). ISBN 0-19-853789-1. MR1420619.

Zeitouni, O. Random walks in random environment. In Lectures on probability theory and statistics, volume 1837 of Lecture Notes in Math., pp. 189-312. Springer, Berlin (2004). MR2071631. 